\title{
Stellar coronagraphy with a redundant array of telescopes in space:
}

\section{The multiple mask coronagraph}

\author{
C. Aime ${ }^{1}$, R. Soummer ${ }^{1}$, and B. Lopez ${ }^{2}$ \\ 1 UMR 6525 Astrophysique, Faculté des sciences, Université de Nice Sophia Antipolis, Parc Valrose, \\ 06108 Nice Cedex 2, France \\ e-mail: Remi.Soummer@unice.fr \\ 2 Observatoire de la Côte d'Azur, Département Fresnel, UMR 6528, 06304 Nice Cedex 4, France \\ e-mail: lopez@obs-nice.fr
}

Received 25 October 2000 / Accepted 7 February 2001

\begin{abstract}
We describe a coronagraphic technique that can be used with an array of telescopes in space. It generalizes the use of Lyot's Masks and Roddier \& Roddier's Phase Masks (Roddier \& Roddier 1997) to an array of telescopes without the densification of the pupil recently proposed by Labeyrie et al. (1999) and Guyon \& Roddier (1999, 2000). The proposed method consists of using an ensemble of masks set on the principal diffraction peaks of the point spread function. The best efficiency is obtained for a redundant configuration of the apertures. A linear array configuration is proposed for solving the problems of chromatism associated with phase mask coronagraphy and permits image reconstruction via tomographic techniques. A numerical simulation is made for a one-dimensional array of telescopes.
\end{abstract}

Key words. instrumentation: interferometers - methods: observational - techniques: image processing stars: planetary systems

\section{Introduction}

The Darwin (ESA) and the Terrestrial Planet Finder TPF (NASA) are space interferometry projects with similar basic specifications and two scientific objectives. One is to realize a space interferometer which will perform high angular resolution imaging and spectroscopy relevant to general astrophysics (globular cluster cores, protostellar disks, mass loss by evolved star etc.). The other objective is to detect and characterize Earth-like extra-solar planets and to search for spectral signatures suggestive of life. The instrumental requirements for Darwin or TPF depend on the science objectives: general astrophysics or the detection and characterization of Earth-like planets.

The direct detection and study of an earth-like planet orbiting a nearby star is an ambitious observational project. However, innovative studies have shown that such an objective is conceivable (Leger et al. 1996; Mennesson \& Mariotti 1997): spectral signatures of life may be found in the infrared, such as those of $\mathrm{H}_{2} \mathrm{O}$ and $\mathrm{O}_{3}$ (Leger 1993;

Send offprint requests to: C. Aime,

e-mail: Claude.Aime@unice.fr
Léger et al. 1999) or in the visible (Labeyrie et al. 1999; Schneider 1999). Several hundred of dwarf stars are located within $15 \mathrm{pc}$ from our solar system. The instrument must resolve at least the typical separation of 0.05 0.1 arcsec which corresponds to an orbit of $1 \mathrm{AU}$ semi major axis, around a star 20-10 pc away.

The main difficulty for direct detection of dim sources like terrestrial extra-solar planets comes from the brightness ratio between the parent star and the planet. It is of the order of $10^{9}$ in the visible wavelength domain and of the order of $10^{6}$ in the infrared domain. Direct detection of Earth-like planets could only be achieved by removing most of the star diffracted light, which is the aim of the so-called coronagraphic or nulling methods.

The coronagraphy technique was first invented by Lyot $(1930,1939)$ for solar corona observations. Applied to stellar observations, it provides direct imaging capabilities, reducing the star diffracted light in the whole field (Bonneau et al. 1975; Smith \& Terrile 1984; Malbet 1996).

Up to now, several concepts have been proposed, bringing a richness of instrumental ideas about the number and the diameter of the telescopes, the baseline configurations 
and the nulling or coronagraphic methods. The Darwin project is a nulling interferometer based on Mariotti's concept (Mennesson \& Mariotti 1997).

The TPF instrument is not completely defined yet and several concepts are still under study, using coronagraphy or nulling interferometry. The instrument envisioned for TPF consists of an infrared interferometer, operating from 7 microns to 20 microns, with baselines between $75 \mathrm{~m}$ and $200 \mathrm{~m}$ and with either a few large telescopes or many small ones (TPF Science Working Group 1999).

Stellar coronagraphy has been recently improved by Roddier \& Roddier (1997) who proposed the use of $\pi$ phase shifting masks instead of occulting masks and also by Gay \& Rabbia (1996) who proposed and developed the Achromatic Interfero Coronagraph (AIC): an achromatic $\pi$ phase shift is introduced by means of a focusing cat's eye in an arm of a Michelson interferometer (Baudoz et al. 2000a,b,c).

We introduce in this paper the Multiple Mask Coronagraphy technique (MMC), which is a generalization of coronagraphy to the purpose of interferometry. This approach can take the advantage of the Roddier \& Roddier's phase mask.

The paper is organized as follows: Sect. 2 gives an heuristic presentation of coronagraphic techniques and its application to diluted apertures. Coronagraphy with a large monolithic aperture is first presented, and generalized to an array of telescopes. The interest of the use of an array of masks, set on the peaks of the Point Spread Function (PSF) of a redundant array of telescopes is proposed here as an efficient solution to the problem. Section 3 gives a numerical one-dimensional simulation for a redundant linear array of telescopes. Advantages and drawbacks, in particular chromatic problems and image reconstruction are discussed in Sect. 4. Conclusions are given in Sect. 5 .

\section{General considerations on coronagraphic masks adapted to the telescope aperture configuration}

We give here an heuristic presentation of Lyot's coronagraphic technique applied to an array of telescopes. We introduce first the basic method applied to a monolithic aperture and generalize it to diluted apertures. This will lead us to show the fundamental interest of redundant apertures, combined with multiple masks for direct extrasolar planet detection.

\subsection{General equations for coronagraphy}

We recall in this section some well known results about coronagraphy (Malbet 1996) and give the Fourier optics formalism used in this paper. Following Guyon and Roddier notations, four planes are involved in the coronagraphic method, as illustrated in Fig. 1: A (telescope aperture), $\mathbf{B}$ (telescope focus), $\mathbf{C}$ (conjugate plane of the

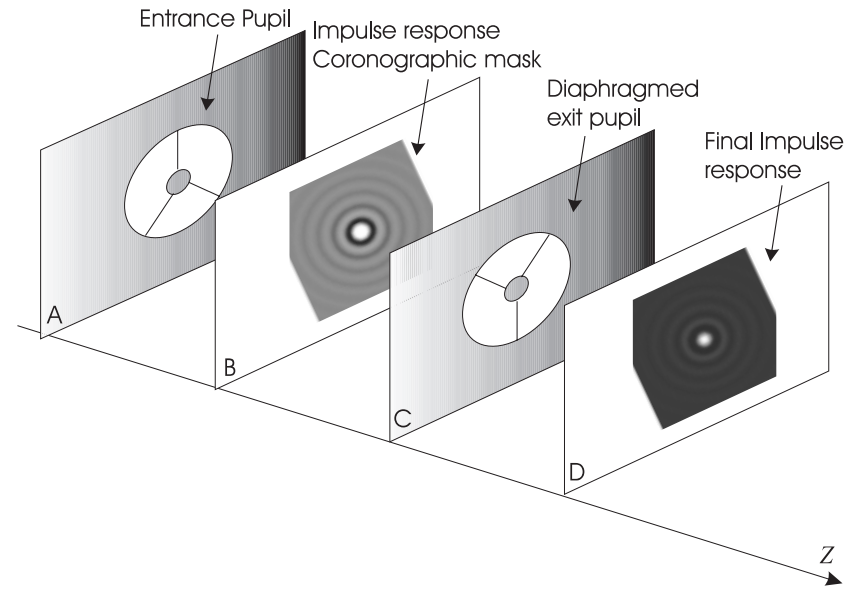

Fig. 1. Illustration of the planes denoted A, B, C and D in the body of the paper. A) telescope aperture, B) telescope focus where the Lyot mask is set. C) image of the entrance pupil where the Lyot's stop (diaphragm) is applied. D) final image plane. Lenses used between planes are not represented

telescope pupil) and $\mathbf{D}$ (plane of the final coronagraphic image).

There is a Fourier transform operation between each of the four planes (from A to B, from B to C and from $\mathrm{C}$ to D). For clarity, we neglect the scaling factors in the notation of the Fourier transforms (convenient units and axes orientation should be used). We denote by $P_{0}(r)$ the actual pupil function in plane A. The amplitude $\Psi_{\mathrm{B}}(r)$ of the wave after the coronagraphic mask of transmission $(1-\varepsilon M(r))$ is proportional to:

$\Psi_{\mathrm{B}}(r)=\widehat{P}_{0}(r)[1-\varepsilon M(r)]=\widehat{P}_{0}(r)-\varepsilon \widehat{P}_{0}(r) M(r)$

where the positive function $M(r)$ describes the central part of the mask: $M(r)$ is 0 outside the mask and 1 inside. $\varepsilon=1$ for Lyot's mask and $\varepsilon=2$ for Roddier \& Roddier's mask.

In plane $\mathrm{C}$ we assume that the Lyot's stop is a diaphragm corresponding to the telescope aperture $P_{0}(r)$. The overall effect is then:

$\Psi_{\mathrm{C}}(r)=\widehat{\Psi}_{\mathrm{B}}(r) P_{0}(r)=P_{0}(r)-\left(\varepsilon P_{0}(r) * \widehat{M}(r)\right) P_{0}(r)$.

For a perfect coronagraphic effect, we would like the quantity $\varepsilon P_{0}(r) * \widehat{M}(r)$, that appears as a subtractive wavefront, to be equal to 1 everywhere within the support of $P_{0}(r)$. This is what we examine in the following sections.

\subsection{Coronagraphy with a monolithic aperture}

Let us assume first that we have a huge monolithic telescope $P_{0}(r)$ of surface $(\Sigma)$. In plane $\mathrm{B}$, the coronagraphic mask set on the core of the Airy disk must have a surface $s$ of the order of $(1 / \Sigma)$. In plane $\mathrm{C}$, its diffraction figure $\widehat{M}(r)$ has a maximum amplitude $s \sim(1 / \Sigma)$ and extends over a surface of the order of $\Sigma$.

In fact, $M(r)$ is smaller than the telescope resolution, so that $\widehat{M}(r)$ extends over a region larger than the telescope aperture $P_{0}(r)$ and may be considered to a first 
approximation as uniform over the aperture domain.

The result of the convolution $\left(P_{0}(r) * \widehat{M}(r)\right)$ is then roughly:

$$
\left(P_{0}(r) * \widehat{M}(r)\right) \sim(1 / \Sigma) \times \Sigma \sim 1 .
$$

The order of magnitude is satisfactory. The term $\varepsilon=2$ (Roddier \& Roddier) allows the use of smaller masks which provide a flatter subtractive wavefront and therefore better cancellation of the star light.

\subsection{Coronagraphy with an array of telescopes}

For the purpose of infrared planet detection, an array of telescopes seems necessary: we assume that $N$ small telescopes of surface $S$ are distributed over the same surface $(\Sigma)$. The overall resolution of this array is thus comparable to that of the previous huge monolithic telescope: the surface of a coronagraphic mask must remain comparable to the instrument resolution $s \sim(1 / \Sigma)$.

In that case, with a single small mask, the coronagraphy is not efficient; indeed, the order of magnitude of the convolution product in plane $\mathrm{C}$ is then:

$$
\left(P_{0}(r) * \widehat{M}(r)\right) \sim(N \times S) / \Sigma=q .
$$

This result is too low by a factor $1 / q=\Sigma /(N \times S)$ where $q$ corresponds to the so-called filling factor (Roddier \& Ridgway 1999). The amplitude in plane $\mathrm{C}$ is therefore not cancelled. Several solutions have been proposed to overcome this problem for interferometric arrays of telescopes.

In the Labeyrie's hypertelescope concept, the pupil, which consists of numerous apertures, is first densified and a single mask can be used (Boccaletti et al. 2000; Labeyrie 1996). The image is directly provided by this densified pupil, to the detriment of the field of view which is reduced in proportion to the densification ratio.

Another solution is proposed by Guyon \& Roddier (2000). The pupil is only densified for coronagraphic purposes and a single phase mask is used. The exit pupil is then rediluted to avoid the field of view limitation.

\subsection{Coronagraphy with a redundant array of telescopes and multiple masks}

The alternative approach we propose in this paper is to consider a redundant configuration of the array of telescopes combined with an array of Lyot or Roddier \& Roddier masks (instead of an unique mask). This makes it possible to recover the factor $1 / q$ so that $\left(P_{0}(r) * \widehat{M}(r)\right)$ remains close to 1 . The masks are set on the maxima of the PSF of the diluted array. We shall see that the best efficiency of this technique is obtained with a redundant configuration.

Let us assume that $P_{0}(r)$ is an array of $N$ telescopes set at regular intervals as shown in Fig. 2. We denote by $d$ the separation between the telescopes. To cover the surface $(\Sigma)$ with these $N$ telescopes, we have approximately:

$\Sigma \sim\left(N \times d^{2}\right)$.

In plane $\mathrm{B}$, the amplitude $\Psi_{\mathrm{B}}(r)$ shows many peaks of surface $\sim(1 / \Sigma)$ each, at separation $(1 / d)$, within the diffraction envelope of surface $\sim(1 / S)$ due to the elementary apertures $S$ (Fig. 2). Multiple masks are used: a coronagraphic mask is set on each of these principal diffracted peaks. Each mask has a surface $\sim(1 / \Sigma)$ and covers a fraction of the peak surface. The number $K$ of masks to use is of the order of the number of peaks within the first diffraction envelope of the elementary apertures $S$ :

$K \sim\left(\frac{1 / S}{1 / d^{2}}\right) \sim\left(d^{2} / S\right) \sim(\Sigma /(N S))$.

In this case, the mask function $M(r)$ describes the positions and shape of the array of masks. The Fourier transform $\widehat{M}(r)$ therefore consists of peaks of amplitude $\left(K \times\left(\frac{1}{\Sigma}\right)\right)$, with separation $d$. These peaks are within the envelope corresponding to a single mask diffraction pattern, which is of the order of $(\Sigma)$ or somewhat larger, since each mask is smaller than the array resolution.

If the pupil is periodic, $\widehat{M}(r)$ will roughly ressemble the pupil function itself, at least for the main features, with peaks at separation $d$ corresponding to the actual apertures. We can write:

$\left(P_{0}(r) * \widehat{M}(r)\right) \sim\left(\frac{K}{\Sigma}\right) \times(N S) \sim 1$

and the dilution factor $1 / q$ of relation 4 is compensated for by the use of multiple masks.

The best result is obtained for a redundant aperture. If the telescope configuration is not redundant, the elementary apertures will not correspond to the peaks of $\widehat{M}(r)$ and the efficiency of the convolution will be reduced. One dimensional numerical simulations for redundant and non redundant apertures are given in the next section.

\section{Numerical simulations for one-dimensional apertures}

We present in this section a one-dimensional illustration of the technique. We briefly give the results for a monolithic aperture and then generalize it to diluted apertures with multiple masks. The notations used are similar to that of Sect. 2, in which we substitute the one-dimensional variable $x$ to the two-dimensional one, $r$. The numerical simulations are made using Mathematica (Wolfram 1999).

\subsection{One-dimensional monolithic aperture}

Replacing $P_{0}(r)$ by $\Pi\left(\frac{x}{\mathcal{L}}\right)$, where $\Pi(x)$ is the function equal to 1 for $|x| \leq 1 / 2$ and equal to 0 elsewhere, Eq. (2) becomes:

$\Psi_{\mathrm{C}}(x)=\left[\Pi\left(\frac{x}{\mathcal{L}}\right)-\varepsilon \times \Pi\left(\frac{x}{\mathcal{L}}\right) *\left(\frac{\sin \pi a x}{\pi x}\right)\right] \times \Pi\left(\frac{x}{\mathcal{L}}\right)$. 
Plane A

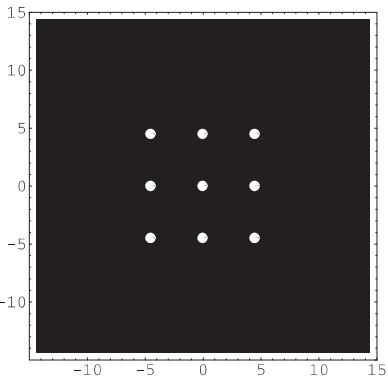

Plane B

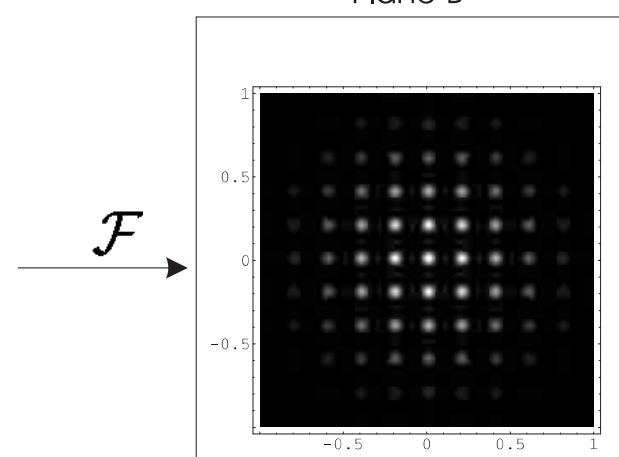

\section{Multiplied by}

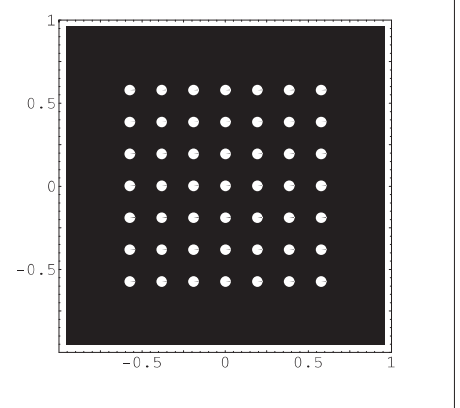

Plane C

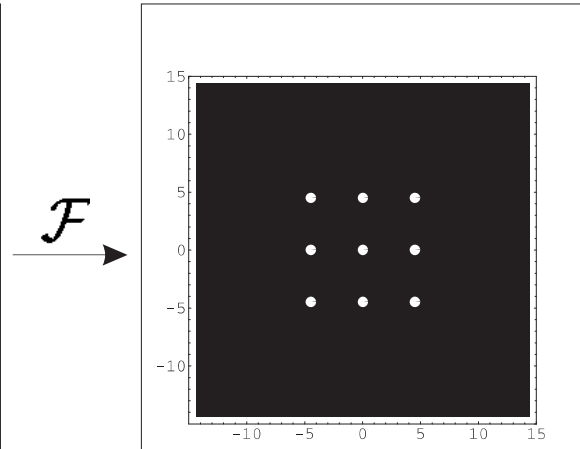

Convolved by

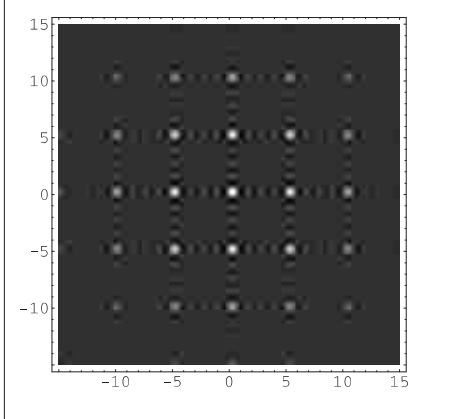

Fig. 2. The planes A, B, C; the symbol $\mathcal{F}$ denotes the Fourier transform. Top left: redundant pupil of 9 telescopes of diameter $\mathcal{D}=1$, with separation $d=5$. Top center: the PSF in plane B shows an ensemble of peaks of separation $1 / d=0.2$, that spread over a region of diameter $1 / \mathcal{D}=1$. Top right: image of the pupil (when the coronagraphic multiple mask is not used). Bottom center: the array of masks is set on the principal diffraction peaks of the PSF: the illustration shows the function $M(r)$, the mask transmission is $1-\varepsilon M(r)$, see Eq. (1). Bottom right: the Fourier transform of the mask function $\widehat{M}(r)$ presents a periodic structure with a separation of the peaks equal to the telescope separation $d=5$. In plane $\mathrm{B}$, the product is applied between the wave amplitude and the masks transmission function. It corresponds in plane $\mathrm{C}$ to a convolution product between their Fourier transform

We can obtain an analytic expression for $\Psi_{\mathrm{C}}(x)$ making use of the Sinus Integral function defined by:

$\operatorname{Si}(x)=\int_{0}^{x} \frac{\sin t}{t} \mathrm{~d} t$

to express the convolution product in relation 8 . Then $\Psi_{\mathrm{C}}(x)$ may be rewritten as:

$$
\begin{aligned}
\Psi_{\mathrm{C}}(x)= & {\left[\Pi\left(\frac{x}{\mathcal{L}}\right)-\frac{\varepsilon}{\pi}(\operatorname{Si}[\pi a(x+\mathcal{L} / 2)]\right.} \\
& -\operatorname{Si}[\pi a(x-\mathcal{L} / 2)])] \times \Pi\left(\frac{x}{\mathcal{L}}\right) .
\end{aligned}
$$

This expression may be useful for an analytical study of a slit aperture telescope and it may be straightforwardly generalized to a two dimensional rectangular aperture.

\subsection{One-dimensional fully redundant array of telescopes}

We now consider that the telescope is an array of $N$ redundant apertures, each of diameter $L$, with a separation $d$ between aperture centers. The overall array length is: $\mathcal{L}=(N-1) d+L$.
The aperture transmission may be written as a sum of shifted elementary apertures:

$P_{0}(x)=\sum_{k=1}^{N} \Pi\left(\frac{x-k d+(N+1) d / 2}{L}\right)$

The constant shift $(N+1) d / 2$ is used to center the overall aperture.

The corresponding amplitude in the focal plane B takes the form of a grating diffraction pattern, with peaks at multiples of $1 / d$, that spread within the diffraction pattern of a single aperture.

$\widehat{P}_{0}(x)=\left(\frac{\sin (\pi L x)}{(\pi x)}\right)\left(\frac{\sin (\pi N d x)}{\sin (\pi d x)}\right)$.

Multiple Lyot's or Roddier \& Roddier's masks are then set on the peaks of $\widehat{P}_{0}(x)$, as explained in Sect. 2.2, to obtain the amplitude $\Psi_{\mathrm{B}}(x)$. Assuming that we use an odd number $K$ of masks, we can write:

$$
\begin{aligned}
\Psi_{\mathrm{B}}(x)= & \left(\frac{\sin (\pi L x)}{(\pi x)}\right)\left(\frac{\sin (\pi N d x)}{\sin (\pi d x)}\right) \\
& \times\left(1-\varepsilon \times \sum_{k=-(K-1) / 2}^{(K-1) / 2} \Pi\left(\frac{x-k / d}{a}\right)\right)
\end{aligned}
$$




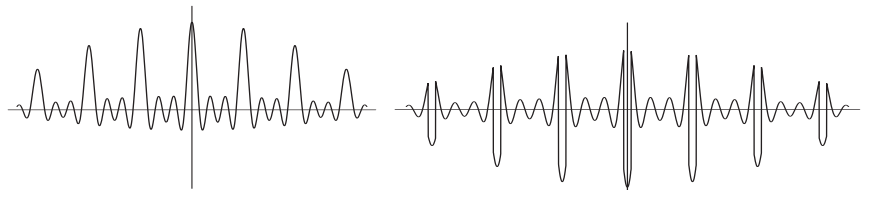

Fig. 3. Amplitude in plane B before and after the application of the $\pi$ phase masks, for an odd number of telescopes $(N=7)$

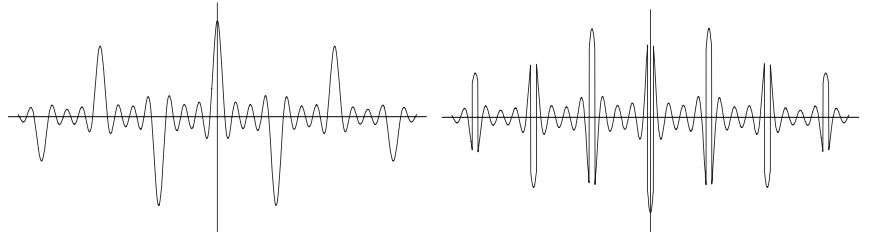

Fig. 4. Amplitude in plane B before and after the application of the $\pi$ phase masks, for an even number of telescopes $(N=8)$

where we recall that $\varepsilon=1$ or 2 according to the type of masks (Roddier \& Roddier's or Lyot's), and $a$ is the width of the masks. An illustration of $\widehat{P}_{0}(x)$ and $\Psi_{\mathrm{B}}(x)$ is made for a redundant array of $N=7$ (Fig. 3) and $N=8$ telescopes (Fig. 4).

In plane $\mathrm{C}$ the expression of the wave becomes:

$\Psi_{\mathrm{C}}(x)=P_{0}(x)-\varepsilon P_{0}(x) *\left[\frac{\sin (\pi a x)}{(\pi x)} \frac{\sin (K \pi x / d)}{\sin (\pi x / d)}\right]$.

This amplitude consists of the subtraction of two terms: the first one is the pupil itself, and the second one a coronagraphic term. Illustrations are shown in Fig. 5 for an array of 7 telescopes for Roddier \& Roddier's Masks.

A second mask is then set in plane $\mathrm{C}$, assumed equal to the entrance aperture and forms $\Psi_{\mathrm{C}}^{\prime}(x)$ :

$\Psi_{\mathrm{C}}^{\prime}(x)=\Psi_{\mathrm{C}}(x) \times \sum_{k=1}^{N} \Pi\left(\frac{x-k d+(N+1) d / 2}{L}\right)$.

A simulation of the amplitude at the exit pupil is shown in Fig. 6. In plane D, the final image of an unresolved star can be written as the quantity $\left|\Psi_{\mathrm{D}}(x)\right|^{2}$. A numerical simulation is given in Fig. 7 .

\subsection{One-dimensional partially redundant array of telescopes}

With a partially redundant configuration, the MMC technique remains usable (with a reduced efficiency), as long as the amplitude in plane B presents sharp periodic peaks to set the multiple masks on. A simulation for a partially redundant aperture (elementary telescopes separated by distances proportional to $(1,3,2,7,1,1)$ is given in Figs. 8 and 9 . The computation is made for Roddier \& Roddier's Phase Masks. Although the coronagraphic term $\varepsilon P_{0}(x) * \widehat{M}(x)$ still operates, the result is not as good as for a fully redundant aperture.

For a non redundant array, in which the separation between apertures is not a multiple of a common value, the intensity in plane $\mathrm{B}$ does not show periodic peaks (Fig. 10), and the MMC technique cannot be applied.

\section{Advantages and drawbacks of the technique}

\subsection{Phase mask chromatism and size optimization}

Phase masks coronagraphy suffers from two main drawbacks: the precision of the phase shift and the sensitivity to the mask dimension, as explained by Roddier \& Roddier (1997).

The extinction capability strongly depends on the precision of the $\pi$ phase shift: Guyon \& Roddier (2000) have shown that if a phase error $\delta \phi$ is introduced by the phase mask, the leaking flux fraction is proportional to $\delta \phi^{2}$, assuming a total extinction of the amplitude inside the exit pupil with optimized entrance pupil apodization.

If the phase mask is made with a plate of refractive index $n$ or with a $\lambda / 4$ shifted back mirror, the phase shift is intrinsically chromatic. This is a problem for broad band observations (from $5 \mu \mathrm{m}$ to $15 \mu \mathrm{m}$ for atmosphere characterization with $\mathrm{TPF})$.

Phase mask coronagraphy is also more sensitive to a variation of the mask size compared to Lyot's coronagraphy, as shown in Fig. 11 for a redundant linear array without apodization.

These results are similar for a monolithic aperture and for a redundant linear array with multiple masks.

For a one dimensional monolithic aperture there is a single optimal diameter for the Phase mask: $a_{\mathrm{PM}}=0.27$ first diffraction lobe. For the Lyot mask, there are several minima: $a_{\text {Lyot }}=0.64 ; 1.62 ; 2.61 ; 3.61$. Note that the minima do not correspond to the zeros of the diffraction pattern.

For a one-dimensional redundant linear array, the optimal mask size also depends on the number of masks $K$. As described in Sect. 2.4, the number of peaks depends on the ratio of the separation to the telescope diameter (here $d / L$ for the one dimensional simulation). Since $K$ must be an integer, this ratio $d / L$ must be optimized for an optimal coronagraphic effect (this was not done in the present analysis).

With 13 masks and the same array configuration as in Fig. 5 , we obtain the optimal values:

$a_{\text {Lyot }}=0.68 ; 1.66 ; 2.69$ and $a_{\mathrm{PM}}=0.29$.

This greater sensitivity of the Phase Mask compared to the Lyot mask becomes a limitation for broad band observations, since the mask size must be adapted for the wavelength dependent PSF.

\subsection{Linear arrays: An optimal configuration}

The solution we propose in this paper to overcome the above problems is to consider a linear configuration of telescopes. In this case, the spatial information is only significant along the array direction and the transverse direction can be used to disperse the light. Illustrations of a dispersed polychromatic PSF for a redundant linear array is shown in Fig. 12. 

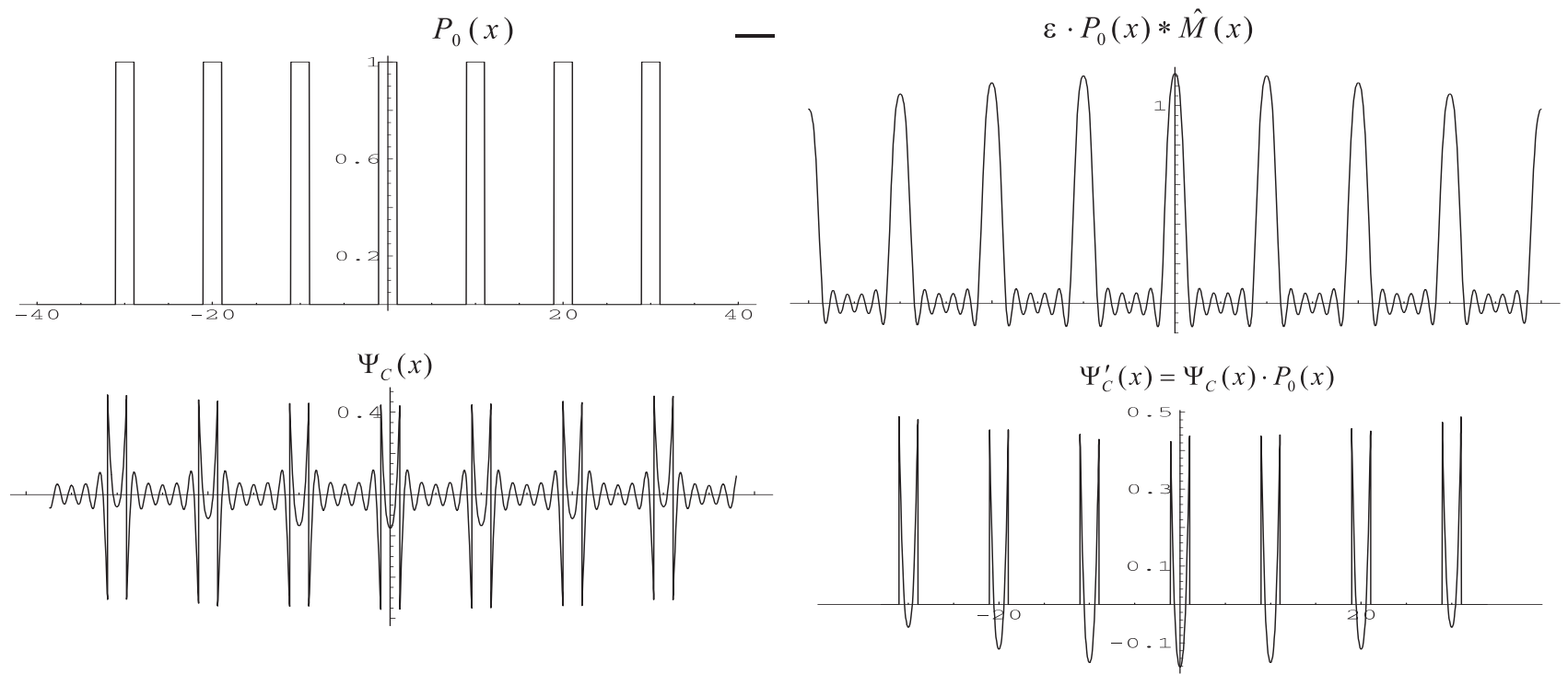

Fig. 5. This figure illustrates the subtraction of the amplitudes in plane C Eq. (14). Top left: the pupil consists of 7 equi-spaced telescopes. Top right: the coronagraphic term $\varepsilon P_{0}(x) * \widehat{M}(x)$ Eq. (14) is computed for $K=13$. Bottom left: the result of the subtraction $\Psi_{\mathrm{C}}(x)$, the amplitude diffracted outside of the apertures is clearly visible. Bottom right: the exit pupil $\Psi_{\mathrm{C}}^{\prime}(x)$ after the application of the diaphragm in plane C. Both $a$ and $K$ must be optimized for best coronagraphic effect. The figure is given for Roddier \& Roddier's phase masks
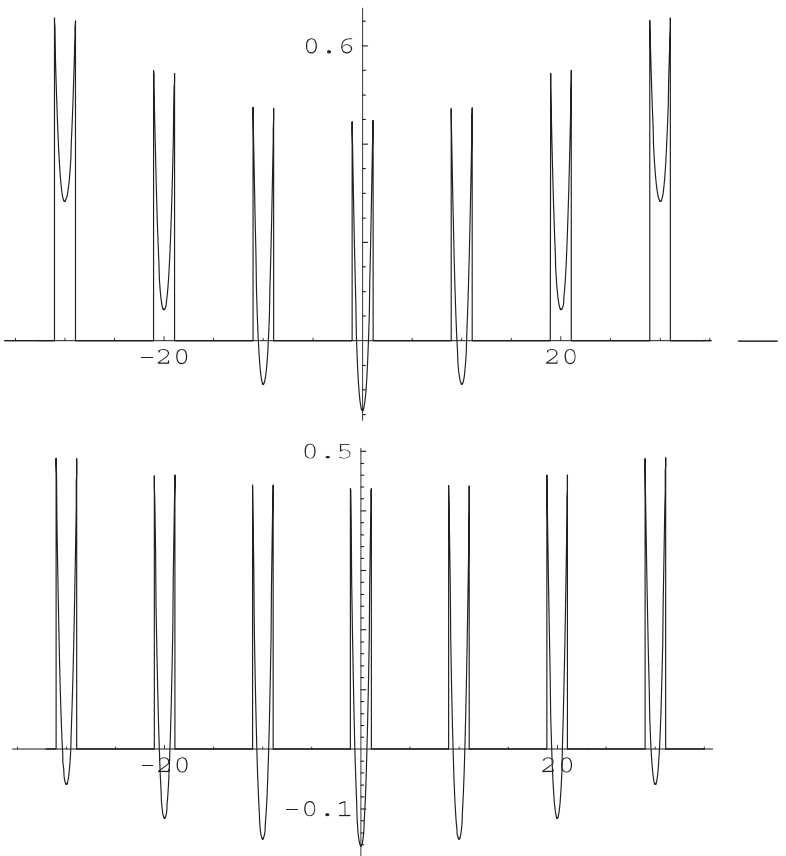

Fig. 6. Exit pupils after the application of the Lyot stop. Top: Lyot's occulting mask. Bottom: Roddier \& Roddier's Phase Mask. The masks size has been optimized in plane B to reduce the residual energy inside the apertures in plane $\mathrm{C}$. The overall residual curvature visible from an aperture to another is due to the width of the mask (enveloppe of the coronagraphic term, see Eq. (14)) whereas the curvature inside each aperture depends on the number of masks used

The coronagraphic masks may then be applied after the dispersion of the light. With such a linear configuration, it seems easier to overcome the chromatism

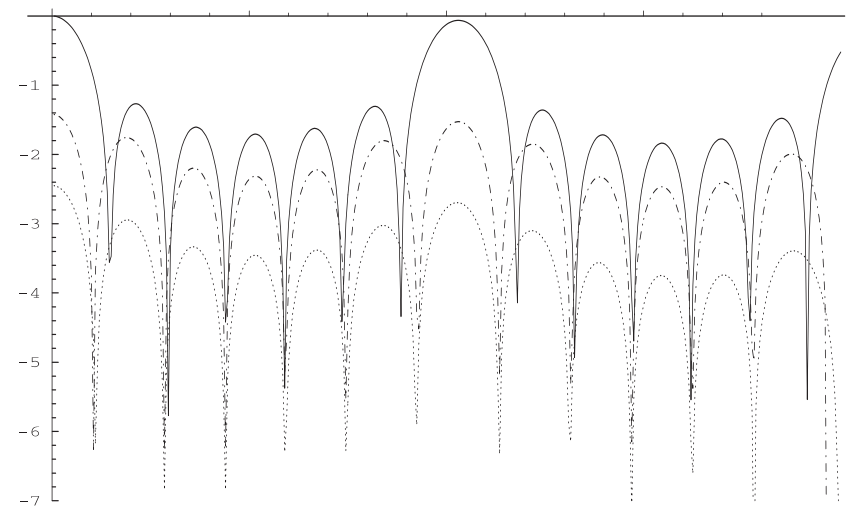

Fig. 7. Light intensity in plane D (Logarithmic scale) for the 7 aperture array and 13 masks. Full line: without coronagraphy. Dashed line: with Lyot's masks. Dotted line: with Roddier \& Roddier's masks

problems. The phase masks can be adapted for each wavelength. A mirror reflection phase mask may be used after the light dispersion, as illustrated in Fig. 12:

The width of the masks is optimized and proportional to $\lambda$, and the thickness (or depth) is equal to $\lambda / 4$.

However, in this presentation, we have not considered the transverse dimension of the entrance pupil and an additional phase error exits: in the $(x, \lambda)$ plane, a monochromatic image will be spread over a finite width in the $\lambda$ direction, corresponding to the spectroscopic limitations. Although several limitations still remain with phase mask techniques, a linear array may provide a favorable configuration to solve the chromatism problems. 

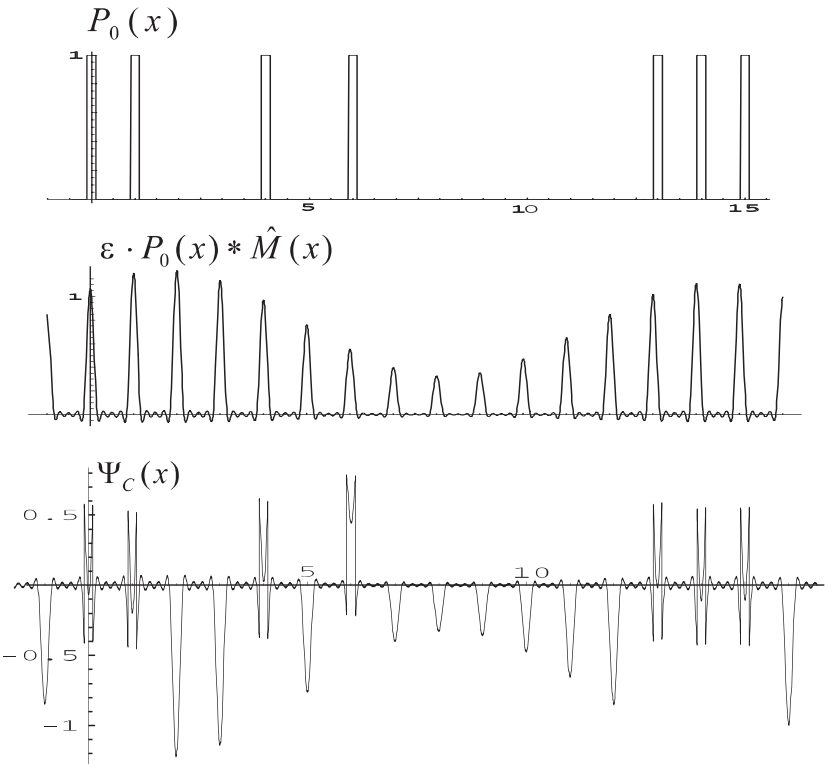

Fig. 8. Use of the MMC technique with a partially redundant array. Top: the pupil is made of 7 apertures with separations proportional to $(1,3,2,7,1,1)$. Center: coronagraphic term. bottom: resulting amplitude in plane $\mathrm{C}$ before the application of the Lyot's stop (see Fig. 5 for comparison)
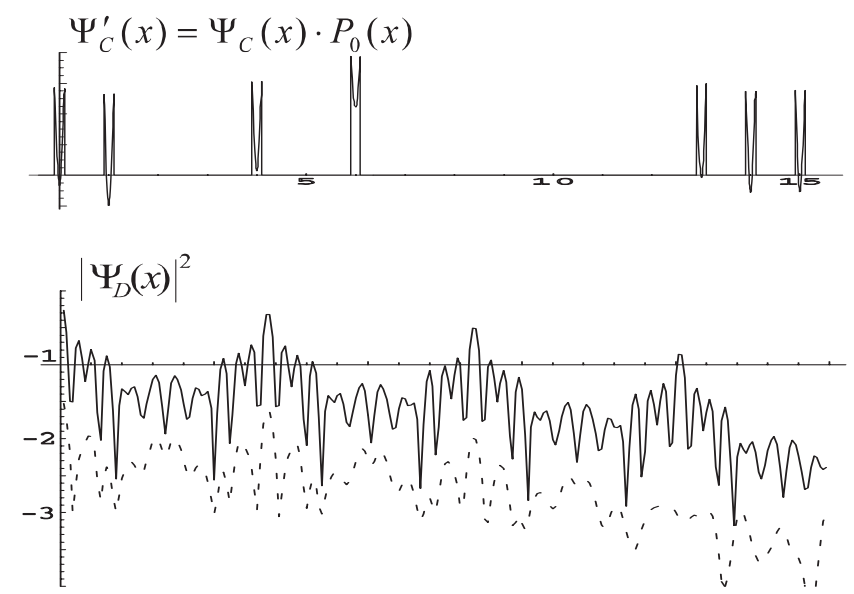

Fig. 9. Top: resulting amplitude $\Psi_{\mathrm{C}}^{\prime}(x)$ after application of the Lyot's stop for the 7 apertures of Fig. 8. The result is fair but for the $4^{\text {th }}$ elementary telescope aperture. If such an aperture configuration were to be used this aperture would have to be occulted by the Lyot's stop. Bottom: resulting intensity in plane D (dotted line), compared to the intensity without MMC (full line)

\subsection{Transmission map}

The residual flux for an off-axis unresolved source has been studied by Guyon \& Roddier (2000) for a densified aperture with phase mask. We obtain the same expression at the origin (from 0 to 2 marcsec) from our simulations with a redundant linear array and MMC as shown in Fig. 13 (just replacing the telescope diameter by the array baseline).

$F_{\mathrm{R}}=C+\left(\frac{8 B}{5 \lambda}\right) \times \theta^{2}$
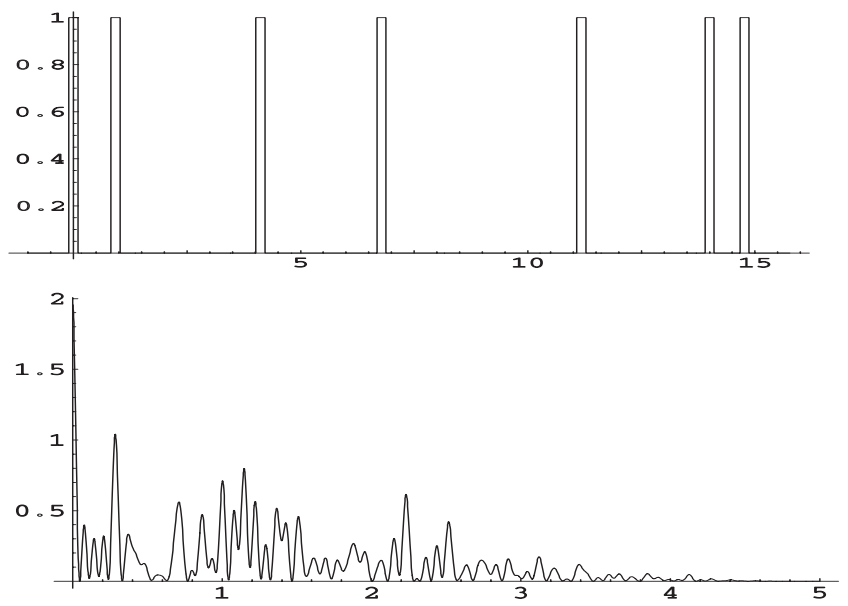

Fig. 10. Top: this figure illustrates the impossibility of using the MMC technique with a fully non redundant pupil in which no separation is a multiple of an other one. Bottom: the PSF of the array does not show identifiable periodic peaks to set the multiple masks

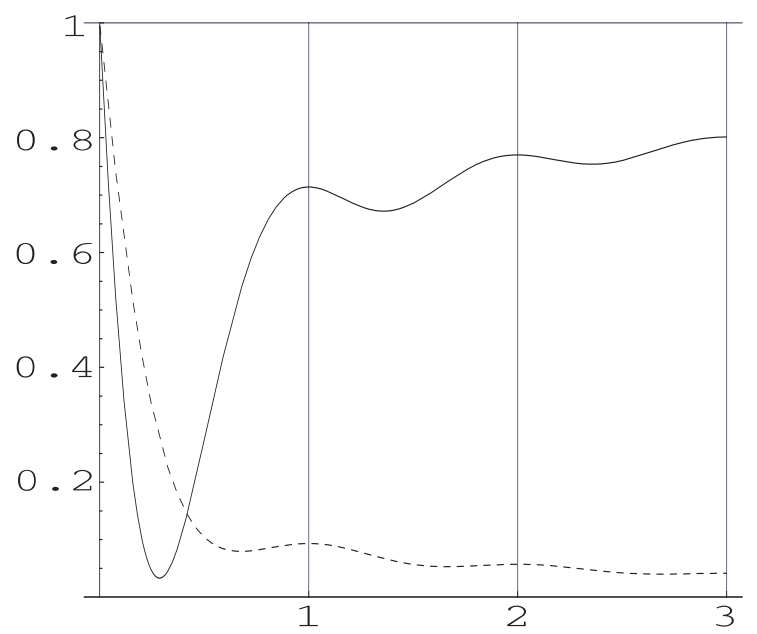

Fig. 11. Normalized residual energy in the exit pupil as a function of the mask diameter in units of PSF dark rings for a redundant linear array of 7 telescopes with multiple masks. Comparison for multiple Phase masks and multiple Lyot masks, with Lyot stop identical to the entrance pupil (no apodization). The Phase mask technique is more sensitive to a variation of the mask size. The minimum of residual energy inside the exit pupil as a function of the mask size is sharper for Roddier \& Roddier's technique (full line) than for Lyot mask (dashed line)

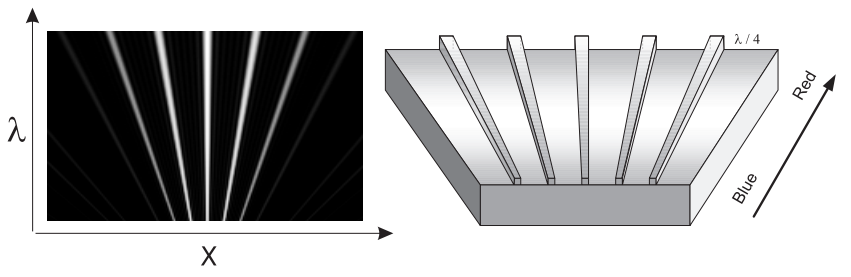

Fig. 12. Left: illustration of the $(X, \lambda)$ dispersed polychromatic PSF for the same array, using cylindrical optics and a spectroscopic device. Right: schematic view of possible reflection phase masks. The width of the masks is proportional to the wavelength and the thickness is $\lambda / 4$ 


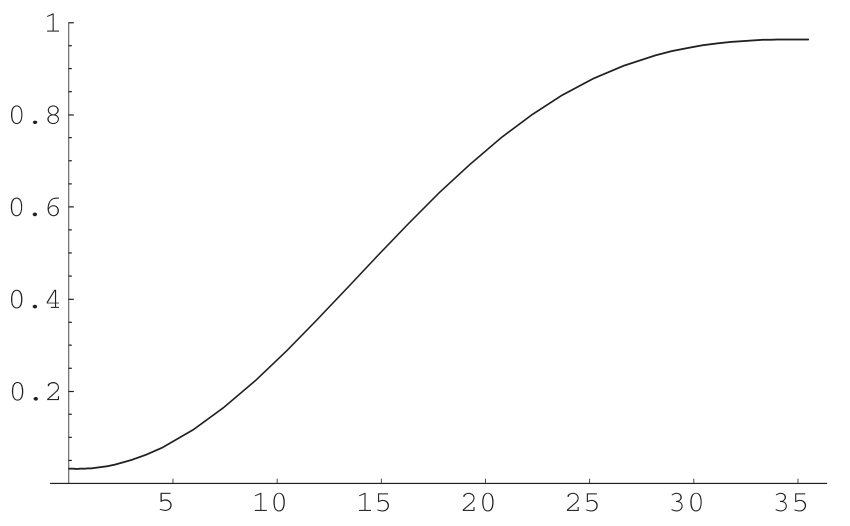

Fig. 13. Overall residual flux for an off-axis unresolved source (transmission map) as a function of the angular position $\theta$ in marcsec ( 7 telescopes, $d=2.5 \mathrm{~m}$, baseline $=70 \mathrm{~m}, \lambda=10 \mu \mathrm{m}$ ). A polynomial function is fitted to the 70 computed points in this simulation

where $B$ denotes the baseline of the interferometer, $\theta$ the angular position of the source and $\mathrm{C}$ is a constant at origin. Using apodized apertures, this constant should approach zero. This is another limitation of the phase mask technique: an additional leaking flux proportional to $\theta^{2}$ exits if the on-axis star is resolved (a typical Sun at $10 \mathrm{pc}$ has an angular size of $\sim 1$ marcsec). This behaviour in $\theta^{2}$ might be considered as insufficient for a nulling interferometer. However, the coronagraphic technique leads to a true image and does not require the same extinction factor, because the signal is not integrated. Indeed, considering Fig. 7, the residual stellar flux at a possible planet position is already of $10^{-3}$ to $10^{-4}$, depending on the exact position. First attempts we have made with apodization (not given here) show that a factor of $10^{-6}$ may be obtained. However, it does not seem interesting to go deeper into our one-dimensional simulation. A more realistic twodimensional simulation combined with apodization would be preferable and remains to be done.

\subsection{Multiwavelength image reconstruction}

For a given number of telescopes, the resolution is optimized in one direction for a linear array. A very good coverage of the $2 \mathrm{D}$ frequency plane may be obtained during the rotation (at least for concentric circles) and a tomographic reconstruction of a two dimensional image is possible (Touma et al. 1995; Martin et al. 1987). The principle of the image reconstruction consists of the inversion of the set of projections of the object (Radon transform) given by the interferometer during its rotation. This technique may profit from the immense work developed for tomographic medical imaging (Barret 1984; Deans 1983).

An example of what could be obtained with a rotating linear array is shown in Fig. 14. The jitter of the rotation axis is a problem which has been already studied and overcome (Touma et al. 1992a,b). The tomographic reconstruction method seems perfectly adapted to the analysis

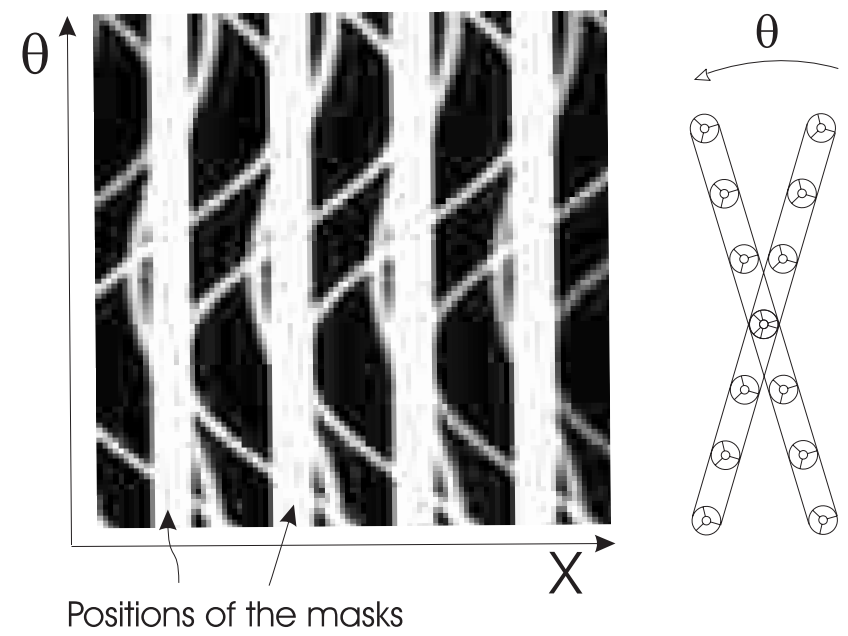

Fig. 14. Set of projections obtained by rotating the linear array of telescopes (Radon plane or sinogram). This monochromatic simulation is made for two planets orbiting around a star. The white stripes correspond to the forbidden zones of the masks

of complex images like multiple planetary systems with exo-zodiacal dust disks.

The redundant configuration of the telescope is necessary for best coronagraphic efficiency. As a drawback, the best frequency coverage that may be needed for general astrophysics purpose is not obtained. However, a minor reconfiguration of the array may break the redundancy and may allow to switch from the planet observation mode with MMC, to the general astrophysics imaging mode. A trivial example for a 4 aperture array is to shift from a $(2,2,2)$ redundant configuration to the well known $(1,3$, 2 ) configuration, just by moving the second telescope.

\section{Conclusion}

We have described in this paper a technique called MMC that permits the implementation of stellar coronagraphy with a space based array of telescopes. An original aspect of the technique is that it does not require the use of densified pupils, unlike other recently-proposed techniques.

The MMC technique is similar to Lyot's coronagraphy, but differs in the fact that multiple masks are set on the main diffraction peaks of the PSF. This array of masks is necessary to remove the stellar light in the whole field. Instead of using Lyot's opaque masks, the technique can take advantage of $\pi$ phase masks proposed by Roddier and Roddier. A redundant configuration of the telescopes is necessary for best coronagraphic efficiency.

The present paper is mainly concerned with the basic presentation of the MMC technique, and several important points have not been developed in this preliminary study.

The numerical simulation made for a one-dimensional aperture is very simple. A more realistic two-dimensional 
simulation, in which all the parameters of the coronagraphic device could be taken into account and optimized, is certainly needed. Our simple simulation however shows that the star light extinction factor is similar to that obtained with a full aperture.

It will be of great interest to use the apodization of the entrance aperture proposed by Guyon \& Roddier (2000). The apodization may compensate the residual curvature of the amplitude inside the apertures and from one aperture to another. This can greatly improve the coronagraphy efficiency, at the expense of a small loss of resolution and a reduction of the transmitted light for the planet. This apodization must be considered in terms of maximization of SNR.

At the moment, our proposed concept for TPF/Darwin would be to consider a rotating redundant linear array coupled with MMC using Roddier \& Roddier phase masks and apodization. The linear configuration is necessary to solve the chromatism problems by means of transverse dispersion.

Three acquisition modes are possible with such a configuration: a multiwavelength image reconstruction is achievable by rotation of the array using robust tomographic methods. However, two acquisitions in orthogonal directions are sufficient for planet detection and positioning. Once the planet is detected, the linear array may be orientated in the planet direction for optimized resolution and spectroscopic acquisition. This mode allows the very long observing times required for atmosphere characterization.

Acknowledgements. The authors would like to thank Steve Ridgway, Olivier Guyon and the TPF Boeing/SVS Science Group for stimulating discussions. Thanks are also due to the referee Pierre Baudoz for constructive comments.

\section{References}

Barret, H. 1984, The Radon Transform and its Applications, vol. XXI (E. Wolf edition)

Baudoz, P., Rabbia, Y., \& Gay, J. 2000a, A\&AS, 141, 319

Baudoz, P., Rabbia, Y., \& Gay, J. 2000b, Proc. SPIE, 4007, 971
Baudoz, P., Rabbia, Y., Gay, J., et al. 2000c, A\&AS, 145, 341

Boccaletti, A., Riand, P., Moutou, C., \& Labeyrie, A. 2000, Icarus, 145, 628

Bonneau, D., Josse, M., \& Labeyrie, A. 1975, in ASSL vol. 54: Image Processing Techniques in Astronomy, 403

Deans, S. 1983, Radon Transform and some of its Applications (Wiley Interscience Edition)

Gay, J., \& Rabbia, Y. 1996, C. R. Acad. Sci. Paris, 322, Serie IIb, 265

Guyon, O., \& Roddier, F. 1999, in ESA SP451 Stockholm, Sweden

Guyon, O., \& Roddier, F. 2000, in Proc. SPIE, vol. 40006

Labeyrie, A. 1996, A\&AS, 118, 517

Labeyrie, A., Schneider, J., Boccaletti, A., et al. 1999, in ESA SP451 Stockholm, Sweden

Leger, A. 1993, The Darwin Mission Concept, proposal to the ESA Horizon 2000 planning process

Leger, A., Mariotti, J., Mennesson, B., et al. 1996, Icarus, 123, 249

Léger, A., Ollivier, M., Altwegg, K., \& Woolf, N. 1999, A\&A, 341,304

Lyot, B. 1930, C. R. Acad. Sci. Paris, 191, 834

Lyot, B. 1939, MNRAS, 99, 580

Malbet, F. 1996, A\&AS, 115, 161

Martin, F., Touma, H., Bijaoui, A., \& Aime, C. 1987, J. Opt., 18,133

Mennesson, B., \& Mariotti, J. 1997, Icarus, 128, 202

Roddier, F., \& Ridgway, S. T. 1999, PASP, 111, 990

Roddier, F., \& Roddier, C. 1997, Astron. Soc. Pac., 109, 815

Schneider, J. 1999, C. R. Acad. Sci. Paris, 327, Serie IIb, 621

Smith, B. A., \& Terrile, R. J. 1984, Science, 226, 1421

Touma, H., Kadiri, S., Martin, F., et al. 1992a, Spectroimagery with a Rotating Slip-Aperture Telescope (RSAT): Cophasing successive projections in the radon plane, Tech. Rep.

Touma, H., Martin, F., \& Aime, C. 1995, Pure Appl. Opt., 4, 685

Touma, H., Martin, F., Kadiri, S., \& Aime, C. 1992b, in Progress in Telescope and Instrumentation Technologies, 527

TPF Science Working Group 1999, The Terrestrial Planet Finder (TPF): A NASA Origins Program to Search for Habitable Planets, ed. C. A. Beichman, N. J. Woolf, \& C. A. Lindensmith, http://tpf.jpl.nasa.gov/

Wolfram, S. 1999, The Mathematica book, Fourth Edition (Cambridge University Press Edition) 